\title{
Development of Multimedia Application for Smartphones
}

\author{
M. A. Mohamed \\ Assoc. Prof. Mansoura \\ University Mansoura \\ Egypt
}

\author{
A. I. Abdel-Fatah \\ Prof. Mansoura University \\ Mansoura \\ Egypt
}

\author{
Bassant M. El-Den \\ Demonstrator Delta University \\ Mansoura \\ Egypt
}

\begin{abstract}
Information security and verify the user's identity has become one of the biggest concerns of institutions and individuals; this interest emerged many types of authentication. Security field uses three different ways; in the oldest documentation of these methods depending on the specific information such as a password or name. The second type depends on something you have his key, such as a smart cards. The last of these species depends on the dynamic qualities for biometry, which measures the properties of natural or behavioral uniqueness in people to document or rooting their identification and verification including famous biometrics (e.g. fingerprint; retina; iris, as well as some facial characteristics and features), also includes personal behaviors (e.g. signature; voice, and walk). All of this biometrics has its advantages that are compatible with the requirements of the different security systems.
\end{abstract}

The proposed implementation model has come as an idea, since today's mobile phones are computers in medium. They are used in running e-mails; agendas; storing data; markets; etc., providing some approaches of security model based on face recognition and/or fingerprint as biometrics approaches for authentication on mobile phones. A new level of mobile phone user's security approach is presented this has been tested with the database. The algorithms have been first profiled in MATLAB and then implemented on android based smart-phones. The balance between accuracy and computation complexity on the application is an important mission to achieve it. In this paper the face recognition security for mobile phones has been represented and applied for face by using principle Component Analysis (PCA) technique.

\section{General Terms}

Image processing; Pattern Recognition, and Security

\section{Keywords}

Smartphone's, Android, Multimedia, Face Recognition, Software Development

\section{INTRODUCTION}

With the rapid progress of embedded system and wireless network, mobile computing has been popular according to its flexibility and portability. Mobile computing has brought tremendous changes in our lives. As wireless network has been deployed almost everywhere, Smartphone's are used more and more widely.

\subsection{Smartphone's: An Overview}

Smartphone is not only a cell phone but also portable computing platform [1]. These smart devices have been the important tools in society for their abundant functions in communication, entertainment, online office and many other fields [2]. In the future, Smartphone's will be more powerful and have new functionalities; they have the potential to replace desktop computers and laptops [3]. Smartphone's are different devices from the desktop computers, they are embedded systems. Such systems are resource limited compared to common computers. They are battery-driven with small memory and slow embedded processors [4]. Software including operating system runs on such devices should be energy efficient with fewer memory footprints and more optimizations.

\subsection{Android: An Overview}

Every smartphone uses an operating system (e.g. Android, IOS and Windows Phone the same way your computer may use Windows as an operating system. It controls the phone's functions and performs tasks to keep the phone working. Android is one of the emerging leading operating systems for Smartphone's as an open source platform. Many Smartphone's have adopted this platform and more will do so in the future.

\subsection{Biometrics: An Overview}

The computer industry that has made the concept of statistical analysis to most people that the best way to prove the identity of the persons using their unique qualities the biometrics is the science that uses statistical analysis to vital human qualities and the most important uses is in. Why it is important is the fact that the physical and behavioral characteristics to humans cannot be transferred to others nor can people forget not stolen, making some researchers known recipe unique human being password. Face recognition is an application used for identifying a person from a digital image or a video frame. This technology has received much attention because of the broad interdisciplinary nature of interest involved in it. It can be used as a biometric system for authentication of the user, recognize someone at a conference or other large gathering and tag him to identify on social networking sites, recognize people and remember their preferences and peculiarities, etc. [5].

Adapting such kind of systems to mobile devices would potentially benefit because of the portability of mobile devices. Hence, creating a stand-alone mobile application that does face recognition on captured images is an interesting avenue to explore. In this paper attempting to address the issues involved in the transformation and proposes the ways to overcome those [6].

The incorporation of face recognition algorithms in to mobile devices has been a challenging problem due to the constraints on processing power, limited storage of the mobile device, limited network bandwidth and connection instability, privacy and security concerns.

This paper is organized as follows. Section 2 describes the motivation of the work. Section 3 describes the design and the architecture of the proposed system. Section 4 presents the 
environment of implementation development. Section 5 presents the description of dataset. At last, Section 6,7 and 8 offers the result, conclusions and future work.

\section{MOTIVATION}

The motivations of this work in general aims implement android based software system to get over the following challenges and problems: (i) the incorporation of face recognition algorithms into smartphone devices has been a challenging problem; (ii) the driving force behind solving this problem is because of the broad interdisciplinary nature of interest in it, and (iii) the processing power and the limited storage of the smartphone devices, limited network bandwidth and connection instability, privacy and security concerns are the difficulties that need to be solved.

\section{FACE RECOGNITION THE ALGORITHM AND SYSTEM ARCHITECTURE}

Many papers related to the subject of implementation based in the mobile phone face recognition approach were published. Different research related to it was started is in the process of testing in implementation. Different approaches were implemented and described in papers which were researching a face recognition approaches for mobile phones in [7]. The most used are $P C A$, the area of face detection and recognition are very complex subject in the field of computer vision. In any paper that deals with this subject, the very first steps considered are those related to face detection. The overflow of proposed system which discussed in this paper is showing in figure 1.

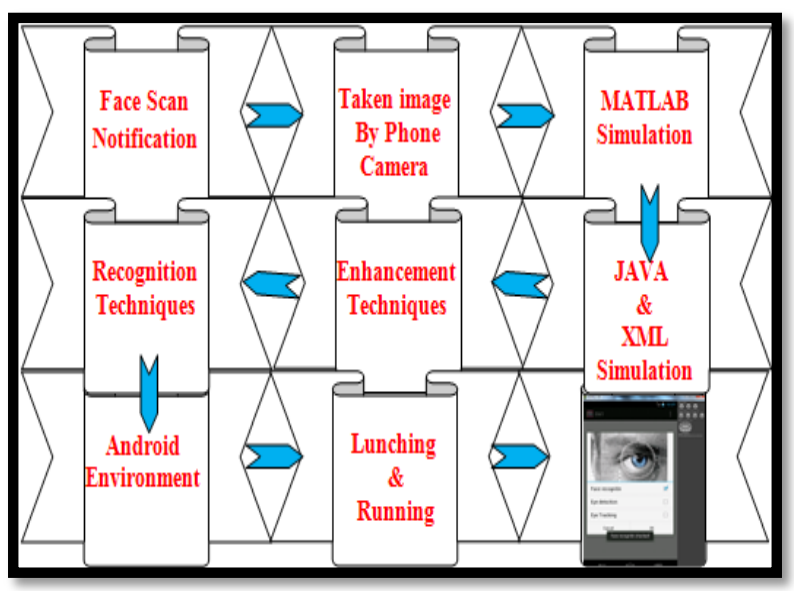

Figure 1. The Architecture of the System

\subsection{Overview}

Recognizing faces is something that people usually do effortlessly and without much conscious thought, yet it has remained a difficult problem in the area of computer vision, where some 20 years of research is just beginning to yield useful technological solutions. As a biometric technology, automated face recognition has a number of desirable properties that are driving research into practical techniques. The problem of face recognition can be stated as identifying an individual from images of the face' and encompasses a number of variations other than the most familiar application of mug shot identification. One notable aspect of face recognition is the broad interdisciplinary nature of the interest in it within computer recognition and pattern recognition; biometrics and security; multimedia processing; psychology and neuroscience. It is a field of research notable for the necessity and the richness of interaction between computer scientists and psychologists.

\subsection{FACE RECOGNITION TECHNIQUES}

The automatic recognition of human faces spans a variety of different technologies. At a highest level, the technologies are best distinguished by the input medium that is used, whether visible light, infra-red [8,9] or three dimensional data [10] from stereo or other range-finding technologies. Thus far, the field has concentrated on still, visible-light, photographic images, often black and white, though much interest is now beginning to be shown in the recognition of faces in color video. Each input medium that is used for face recognition brings robustness to certain conditions, e.g. infra-red face imaging is practically invariant to lighting conditions while three dimensional data in theory is invariant to head pose. Imaging in the visible light spectrum, however, will remain the preeminent domain for research and application of face recognition because of the vast quantity of legacy data and the ubiquity and cheapness of photographic capture equipment [7].

\subsection{Face Recognition System Based on PCA Technique}

The Principle Component Analysis ("PCA") is the common and successful techniques which have been used in image recognition and compression. It is a statistical method among many different factors which are applied in analysis. The main goal of $P C A$ is to reduce the large dimensionality of data, to the smaller dimensionality of feature space, and this needed to describe data economically. This happens when there is a strong correlation between observed variables.

The PCA enable us to do: (i) Prediction; (ii) Redundancy removal; (iii) feature extraction, and (iv) Data compression

Application of face recognition may be applied in Many different areas: (i) face identification; (ii) face classification, and (iii) Biometric security

The idea of implementing and using $P C A$ for face recognition is to express images in one dimension vector of pixels constructed from two dimension image into the compact principle components of the feature space. Automatic face recognition system tries to find the identity of a given face image according to their memory. The memory is simulated by a training set. The training set consists of the features extracted from known face images of different persons. The task is to find the most similar feature vector among the training set to the feature vectors as a given test images. The feature extraction algorithm which has been used in this case is $P C A$ [7-10].

\section{Development Environment}

System consists of two different parts, the server part and the face recognition part as follows:

- Mobile Platform - System is embedded into Samsung Galaxy S4 model of mobile phone and supports JAVA technology and is integrated with JAVA.

- Android Mobile Technology - JAVA SDK and Android technology, with DROID emulator.

- Database - Small database is developed for testing and evaluation. Database consists of face images. 
- Face Recognition Method - Face Recognition part is developed using MATLAB and JAVA. It is integrated with the server.

\section{TRAINING AND TEST SET}

Face 94 database has been used as Database of faces, (formerly 'The ORL Database of Faces'), contains a set of face images taken at the lab Figure 2. Show sample of face database which contains 40 persons, with different pose the files are in PGM format the size of each image is $92 \times 112$ pixels, with 256 grey levels per pixel. Sample of this database have been prepared for testing on ten cases. This is implemented and tested in MATLAB. For some subjects images are taken from different prospective, varying the lighting, facial expressions as: open/closed eyes, smiling/not smiling; facial details: glasses / no glasses. All of the images are taken against a dark homogeneous background with the subject in an upright frontal position with a tolerance for some side movements.

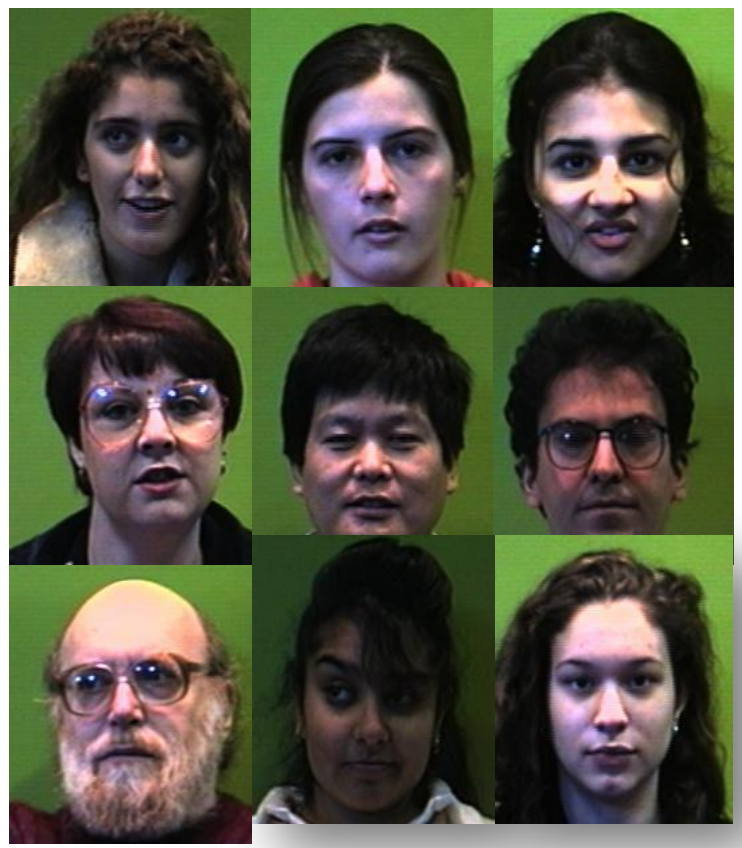

Figure 2: Sample of training database

The database can be retrieved from [11] as a $4.5 \mathrm{Mbyte}$ compressed. Determined the Euclidean distance between test images and training image and have found the closest Euclidian distance. A threshold is set such that if the closest distance above the threshold, the test face is considered unrecognized, and if below, is associated with the identity of the closest face.

\section{IMPLEMENTATION ON DROID}

After profiling and testing the face recognition algorithm in MATLAB, we then implemented the algorithm on the Samsung Galaxy $\mathrm{S}$ model of DROID phone. For the implementing of face detection algorithm it is based on a very robust, assumed using the Viola-Jones. It was used to crop a face bitmap out of the picture taken with the phone camera [12-13].

The development of the face recognition algorithm on the Android platform have found more efficient by using Eigenface, and implemented in Android. The Eigenface projection matrices were computed in Matlab for our fixed training set and stored on the Droid external storage.
The steps previously described for Matlab reproduced: preprocessing, mean removal and projection. This approach Execution particularly by stay close to the MATLAB implementation because the same training set eigenvectors was used [14]. A critical challenge was to keep the computation time as small as possible, so that the user experience is the best possible. In order to achieve that goal, first down-sample the high resolution camera pictures by a factor of 8 . The computation time is then roughly 64 times smaller than for a full size image. Second, the I/O management was critical. Convert the text files written with Matlab into binary files to store our Eigenface and mean face matrices in an efficient way. Then it was possible to read these values with a Data Input Stream, which is much faster than to read line by line from a text file. For example, the projection took only $0.8 \mathrm{sec}$ with a Data Input Stream, compared to $14 \mathrm{sec}$ with text files [15].

\section{RESULTS}

An Android app that captured face images was successfully created from the ground up. Furthermore, the face detection algorithm was executed on Android, as well as shown in Figure. [3-6]. after encountering difficulty integrating face Recognition code with the Android platform, the face recognition algorithm was implemented with MATLAB. The separate components of face recognition and face detection worked successfully independently; however, they were not both integrated together on the Android platform. Since the difficulties of implementing face recognition on an Android device, is solved and now a new area of using it as a security feature for the device.

For the accuracy of the experiments, the training set should not overlap the test set. This paper is a prototype developed to test face recognition on mobile phone. Here, an implemented $P C A$ algorithm for face recognition on MATLAB side, and have developed the JAVA authentication methods for Android.

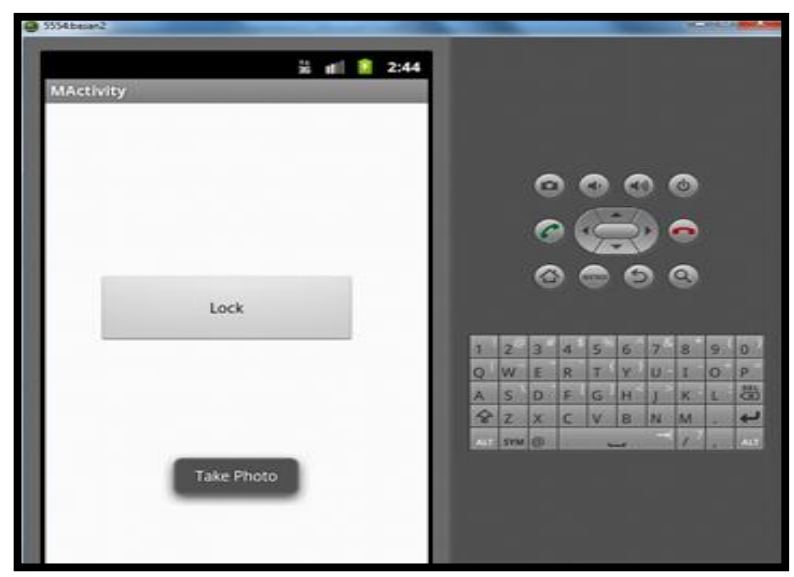

Figure 3: Hint note to the owner of mobile 


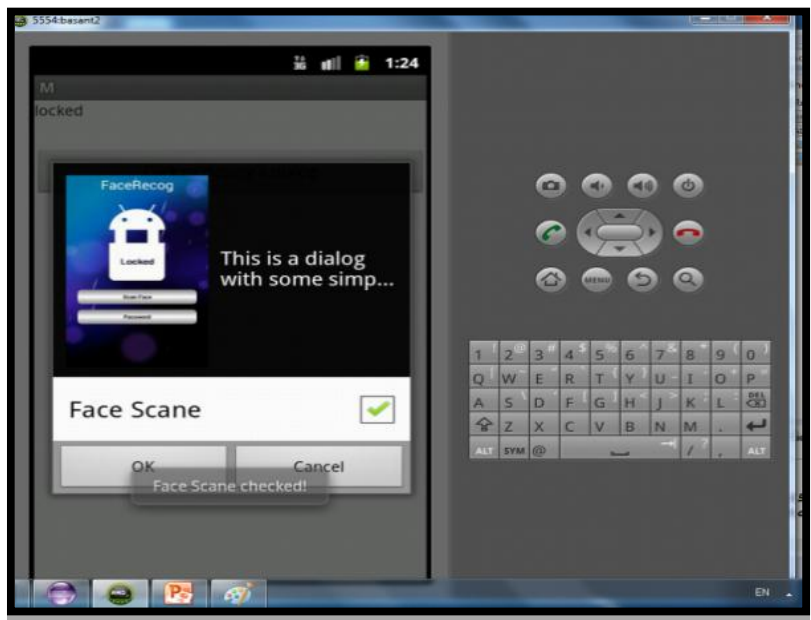

Figure 4: Hint for the Face Scan Application

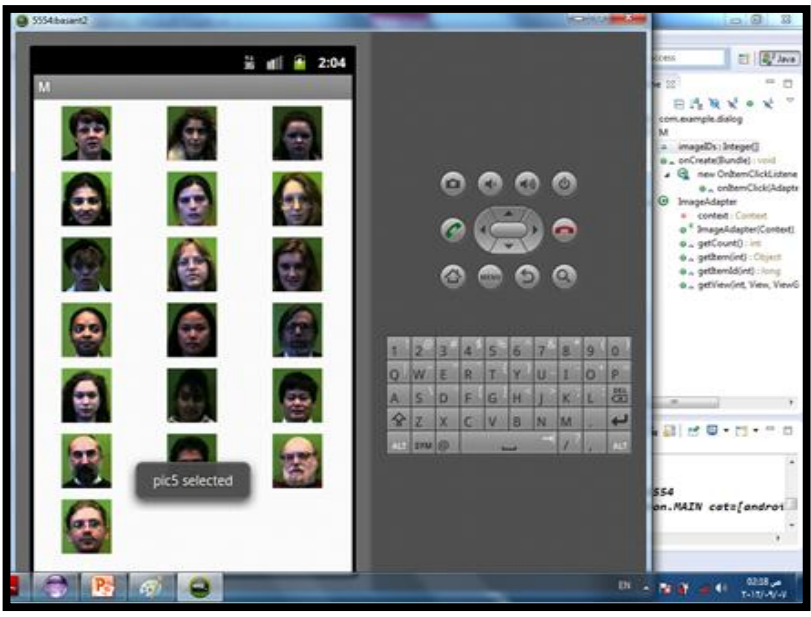

Figure 5: Portion of Data base that is stored on mobile memorv

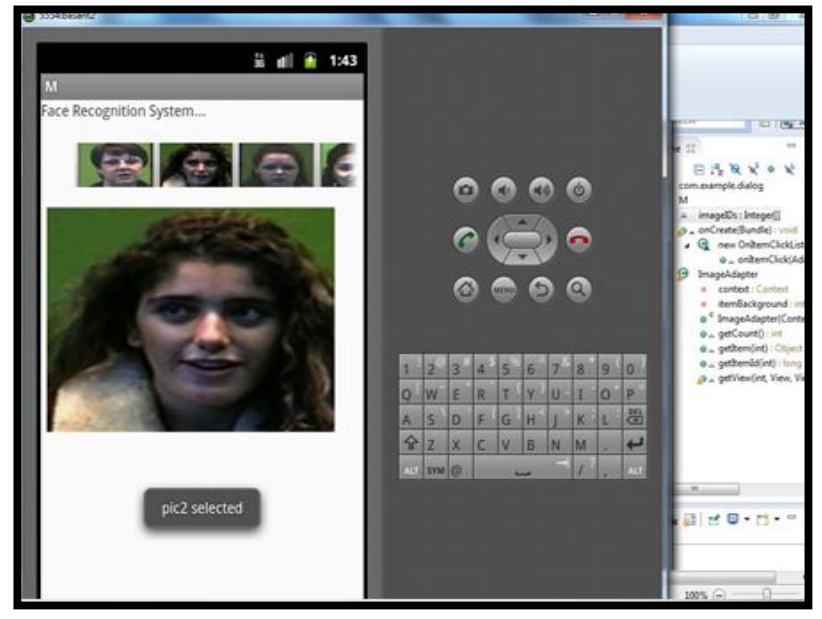

Figure 6: Recognition process is complete for the owner of mobile

\section{CONCLUSION}

In this paper, examination of how can improve the security of a mobile phone, authentication and implemented it. An extensive and exhaustive work in implementing face recognition with mobile phone authentication and making it work in real time on Android Samsung Galaxy S4 has been done.

\section{FUTURE WORK}

$P C A$ is one of the basic algorithms for face recognition and as the database size increases the computation slows down. There are many more efficient and fast algorithms like neural networks, compressed sensing, etc. which can be modified to be able to implement on mobile phones and achieve real time recognition on huge databases. Also providing the option for online training for large databases is another extension.

Even a significant research has been done; there is still open room for continuing working on this subject. With mobile phones usage, traditional method of user authentication changes and raises important security issues. An open question to future work stays in video and recognition subject in mobile phone security as part of mobile authentication.

\section{REFERENCES}

[1] R. Ballagas, J. Borchers, M. Rohs, J.G.Sheridan, "The smart phone: ubiquitous input device", IEEE Pervasive Computing, vol. 5, issue 1, March 2006, pp. 70-77, doi: 10.1109/MPRV.2006.18.

[2] V. Loscri, M. Tropea and S. Marano, "Voice and video telephony services in smartphone", EURASIP Journal on Wireless Communications and Networking, vol. 2006, issue 2, 2006, pp. 16-24, doi: 10.1155/WCN/2006/84945.

[3] L. Tarrataca, A.C. Santos and J.M.P. Cardoso, "The current feasibility of gesture recognition for a smartphone using J2ME", Proc. the 2009 ACM symposium on Applied Computing, 2009, pp. 16421649, doi: 10.1145/1529282.1529652.

[4] S. Kulkarni and P. Agrawal, "Smartphone driven healthcare system for rural communities in developing countries", Proc. of the $2^{\text {nd }}$ International Workshop on Systems and Networking Support for Health Care and Assisted Living Environments, 2008, Article No. 8, doi: $10.1145 / 1515747.1515758$.

[5] N. Ai, Y. Lu and J. Deogun, "The smart phones of tomorrow", ACM SIGBED Review, vol. 5, issue 1, Article No. 16, Special issue on the RTSS forum on deeply embedded real-time computing, 2008, doi: $10.1145 / 1366283.1366299$.

[6] Y. Fei, L. Zhong and N.K. Jha, "An Energy-Aware Framework for Dynamic Software Management in Mobile Computing Systems", ACM Transactions on Embedded Computing Systems, Vol. 7, No. 3, Article 27, April 2008, doi: 10.1145/1347375.1347380.

[7] Sabri M., Nurulhuda I., "Mobile to server face recognition: A System overview," World Academy of Science, Engineering and Technology, vol. 69,pp. 767$771,2010$.

[8] P. Jonathon Phillips, Patrick J. Rauss, and Sandor Z. Der. FERET (Face Recognition Technology) Recognition Algorithm Development and Test Results. Technical Report ARL-TR-995, Army Research Laboratory, October 1996.

[9] F. Prokoski. History, Current Status, and Future of Infrared Identification. In Proceedings of IEEE Workshop on Computer Vision Beyond the Visible Spectrum: Methods and Applications, pages 5-14, June 2000. Facial Thermo gram. 
[10] Chin-Seng Chua, Feng Han, and Yeong-Khing Ho. 3D Human Face Recognition using Point Signature. In International Conference on Face and Gesture Recognition, pages 233-238, 2000.

[11] http://cswww.essex.ac.uk/mv/allfaces/faces94.html Last visit at 05-march-2014.

[12] Sharma M., Singh S., "Practical implementation of matlab based approach for face detection using feed forward network", Journal of Computer Science and Information Security, vol. 9, pp. 284-290, 2011.

[13] L. Ma and K. Khorasani, "Facial expression recognition using constructive feedforward neural networks", IEEE
Transactions on Systems, Man and Cybernetics, Part B, Vol. 34, No. 3, June 2004, pp. 1588-1595.

[14] Choi K., Toh K.A., Byun H., "Real-time training on mobile devices for face recognition application," Elsevier Pattern Recognition, vol. 44, pp. 412-320, 2011.

[15] Sabri M., Nurulhuda I., "Mobile to server face recognition: A System overview," World Academy of Science, Engineering and Technology, vol. 69, pp. 767$771,2010$. 